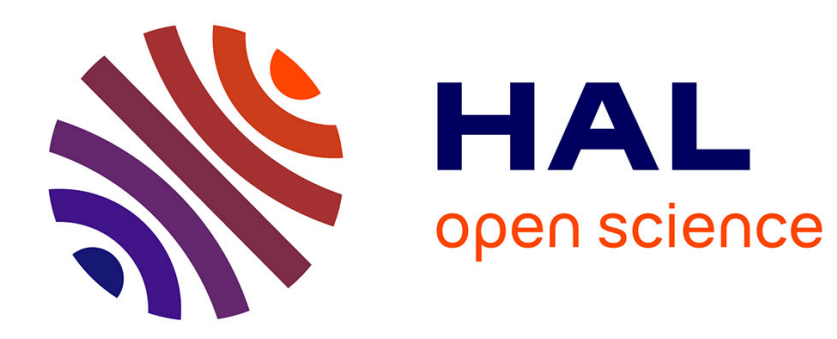

\title{
Les couples gays et la norme d'égalité conjugale Jérôme Courduriès
}

\section{To cite this version:}

Jérôme Courduriès. Les couples gays et la norme d'égalité conjugale. Ethnologie française, 2006, 36 (4), pp.705-711. halshs-00589669

\section{HAL Id: halshs-00589669 https://shs.hal.science/halshs-00589669}

Submitted on 29 Apr 2011

HAL is a multi-disciplinary open access archive for the deposit and dissemination of scientific research documents, whether they are published or not. The documents may come from teaching and research institutions in France or abroad, or from public or private research centers.
L'archive ouverte pluridisciplinaire HAL, est destinée au dépôt et à la diffusion de documents scientifiques de niveau recherche, publiés ou non, émanant des établissements d'enseignement et de recherche français ou étrangers, des laboratoires publics ou privés. 
Les couples gays et la norme d'égalité conjugale

Jérôme Courduriès

jcourduries@gmail.com

\section{RESUME}

En dépit d'une volonté sociale de norme égalitaire, la répartition des rôles dans les couples hétérosexuels reste sexuée et inégalitaire. Qu'en est-il dans les couples homosexuels masculins ? Le modèle hiérarchique des relations entre les sexes traverse tous les couples, affectant négativement des activités : ainsi que les résultats d'une enquête qualitative le montrent, un homme en couple avec un autre homme ne peut se voir assigné sans difficulté à des compétences domestiques et à des rôles traditionnellement perçus comme féminins, donc « dépréciés » et «subalternes ».

Mots-clés: Conjugalité, homosexualité masculine, égalité, travail domestique, sexualité, identité masculine.

Les modèles de référence parmi les hommes homosexuels ont changé depuis les années 1980, époque où le sociologue Michael Pollak décrivait le mode de vie en ghetto et le "marché des échanges sexuels » [Pollak, 1982 : 56-80]. Aujourd'hui, la sphère privée et la vie conjugale constituent une véritable alternative [Adam, 1999; Schiltz, 1998]. Cette évolution et la reconnaissance des couples de même sexe en France nous ont amené à interroger les modalités de la vie conjugale gay. Comme les hétérosexuels, les gays se rencontrent, s'aiment, souvent vivent ensemble, parfois se disputent... Comme les hétérosexuels, ils partagent la norme d'égalité conjugale qui leur paraît encore plus évidente qu'elle ne l'est entre homme et femme, puisque le couple gay n'unit pas deux personnes appartenant à des sexes hiérarchisés.

Toutes les enquêtes menées jusqu'ici auprès de couples hétérosexuels montrent qu'en dépit de la force de la norme égalitaire, la répartition des rôles reste sexuée et inégalitaire, et ce malgré les progrès de l'émancipation féminine et la remise en cause du lien nécessaire entre sexe biologique et rôles sociaux. Qu'en est-il dans les couples homosexuels masculins ? Comment reçoivent-ils la norme d'égalité conjugale ? La différence des sexes ne peut y être convoquée, mais n'existe-t-il pas néanmoins des fonctionnements inégalitaires, en particulier dans les domaines du travail domestique et de la sexualité ? En quoi la gestion de la sexualité dans les couples gays se pose-t-elle de manière spécifique ? La question se pose d'autant plus que les couples d'hommes cumulent sans doute plus souvent que les autres des facteurs d'une possible inégalité : les différences d'âge semblent être plus importantes qu'entre hommes et femmes et elles s'accompagnent souvent de disparités sociales et de revenus ${ }^{1}$. Dès lors, le souci d'égalité dans les différents champs de la vie conjugale se fait encore plus pressant, surtout, il faut bien le dire, pour celui qui est dans une position qu'il juge déficitaire ; et dans bien des cas son partenaire partage le même souci. Comment sont gérées ces inégalités ? C'est ce que nous analyserons en évoquant rapidement la question de l'organisation financière du couple, puis d'une façon plus approfondie, celle des tâches domestiques et enfin celle de la sexualité conjugale.

L’organisation domestique 
C'est dans le domaine financier que les faits s'accordent le plus facilement avec les principes. Les deux compagnons se répartissent les charges, et l'organisation adoptée perdure jusqu'à ce qu'intervienne un changement nécessitant une nouvelle répartition, tel que l'évolution des revenus ou l'achat d'un appartement. Certains couples calculent la part de chacun proportionnellement à son niveau de revenus [Carrington, 1999 : 91-93], ainsi, François, 34 ans, informaticien, et Olivier, 36 ans, ingénieur, qui ont décidé d'acheter ensemble un appartement. Les mensualités de remboursement de leur prêt seront de $1000 €$; ils calculent leur participation individuelle au prorata de leurs revenus, Olivier participant ainsi davantage au financement de leur appartement. Johan, 33 ans, artiste, las de gérer ses découverts vertigineux, a un jour proposé à son compagnon Fabrice, 38 ans, enseignant, une procuration sur son compte. Depuis, Fabrice essaie de couvrir ces découverts en faisant des versements depuis son propre compte, puis lorsque Johan a quelques rentrées d'argent, il se rembourse. Il règle également la plupart de leurs factures communes.

Les couples rencontrés ouvrent très rarement un compte joint, contrairement aux couples hétérosexuels - particulièrement ceux qui sont mariés- et aux couples homosexuels de San Francisco [Carrington, 1999]. Malgré cela, ils mettent en œuvre une organisation financière fondée sur la solidarité - qui semble généralement satisfaire les deux partenaires ${ }^{2}$.

Il est un autre domaine dans la vie conjugale des gays où la norme d'égalité est encore plus forte, mais néanmoins difficile à mettre en œuvre : le travail domestique. De nombreuses études ont montré comment les rapports inégalitaires et hiérarchisés entre les sexes s'inscrivent dans le quotidien conjugal. Durant les trente dernières années, le modèle traditionnel des responsabilités domestiques réservées aux femmes a progressivement laissé la place - dans les discours - à un modèle de conciliation [Kellerhals et al., 2004 : 87]. Toutefois, les femmes continuent d'assumer la plus grande part des tâches domestiques [Anxo et al., 2002 ; Barrère-Maurisson, 2003 : 114-115 ; Filiod, 2003 : 143], le véritable changement résidant dans le fait que les rôles domestiques, immuables jusqu'aux années 1970, deviennent ouverts et négociables au sein de la famille.

Certaines tâches domestiques, telles le bricolage et le jardinage, sont généralement connotées comme masculines, d'autres telles la cuisine, la lessive, le repassage, la vaisselle et le ménage comme féminines, enfin d'autres, telles les courses, la gestion des papiers et de l'argent, sont considérées comme mixtes [Brousse, 2000]. Les hommes s'investissent parfois dans les activités domestiques à dominante féminine, toujours sur le mode du choix [Dussuet, 1997 : 79]. Les femmes continuent massivement de s'occuper du ménage, de la cuisine et du linge [Kaufmann, 1992] et les hommes sont tout au plus des aides. La transmission fortement sexuée des manières ainsi qu'une forme de mémoire féminine des tâches domestiques, entretiennent d'une certaine façon la résistance des hommes au changement. Mais une répartition déséquilibrée des tâches ménagères n'est pas souvent remise en cause par la femme qui, dans un couple, en pâtit et attend en retour que son compagnon, à défaut de faire le ménage, s'engage dans la relation conjugale. Qu'en est-il dans un couple d'hommes ?

Dans son ouvrage sur La domination masculine, Pierre Bourdieu [1998] rappelle que les homosexuels ont été élevés nécessairement comme des hétérosexuels. L'apprentissage des rôles et de la spécialisation dans le travail domestique commence très tôt, et chaque individu reçoit, au fur et à mesure qu'il avance dans la vie, des éléments de définition de l'identité masculine ou féminine ; cela vaut aussi pour les homosexuels. Nos interlocuteurs ont donc fait l'objet d'une socialisation conforme à leur sexe, de sorte qu'ils ont hérité de manières de voir et d'agir propres à celle-ci. Si bien que lorsque deux hommes vivent ensemble, rien, dans leur socialisation, ne semble les prédisposer à un rôle conjugal tourné vers la maison. Nos interlocuteurs sont pris entre deux modèles: d'un côté l'idéal d'un partage équitable, majoritaire dans les discours et renforcé par la mise en présence de deux hommes ; d'un autre 
côté le modèle traditionnel d'une division sexuée des tâches domestiques qui n'incite pas les hommes à prendre massivement part aux tâches ménagères. Un homme, même s'il vit avec un autre homme, ne peut pas aller trop loin dans leur prise en charge, au risque de mettre en péril à ses yeux sa qualité d'homme. Et pourtant le quotidien impose un travail à faire, ne serait-ce que minimal. Comment ces tâches sont-elles assumées?

\section{De la difficulté de décrire l'organisation de son couple}

La question du travail domestique se pose généralement durant les premiers jours de la vie sous le même toit, qui débute souvent rapidement après la rencontre. Discuter des tâches domestiques n'est pas une priorité, chacun étant occupé à faire la connaissance de l'autre. Selon François, cadre commercial, 38 ans, vivant depuis deux ans et demi avec Sylvain, 34 ans, agent administratif, ces répartitions se sont faites « naturellement », sans discussion.

Que signifie alors «naturellement»? Est-ce une façon d'éluder la question, de nier qu'il puisse y avoir un problème? Beaucoup de nos interlocuteurs déclarent, par message électronique ou en face à face, que le partage a été « équitable». Ces réponses systématiques montrent le poids de l'exigence d'équité en ce domaine, que les travaux de Jean-Claude Kaufmann notamment ont mis en évidence. Si les bonnes volontés entrent en action - du moins au début de l'histoire conjugale - les habitudes reprennent vite le dessus. Pourtant les individus croient à cette idée d'égalité entre les sexes au point de ne pas en voir l'échec et de tenir encore un discours égalitaire, en désaccord avec la réalité des faits [Kaufmann, 1992: 231-234]. Ce discours dominant sur l'égalité, les gays en couple le reprennent à leur compte, mais qu'en est-il vraiment?

Ces derniers ne voient pas non plus et ne souhaitent pas voir les modalités concrètes de l'organisation de la vie conjugale [Kaufmann, 1992: 155]. La plupart de nos interlocuteurs n'ignorent pas les arrangements domestiques, ne serait-ce que parce qu'ils vivent, précisément, avec un autre homme. L'illusion du « tout naturellement » leur permet de taire la part de calcul dans les échanges entre conjoints. Ils ne souhaitent pas dévoiler l'organisation matérielle du lien conjugal, qui implique calcul et négociation, car cela les amènerait à objectiver leurs pratiques. Ceux qui éprouvent quelques difficultés avec la gestion du quotidien conjugal y voient un danger : le fait même de dire ses difficultés à un tiers revient à leur donner corps en dehors du couple, ce qui peut être vécu comme une trahison de l'unité conjugale et peut expliquer les hésitations de nos interlocuteurs à s'exprimer sur cet aspect de leur vie à deux.

\section{Un partage équitable et souple}

La répartition des tâches domestiques ne pose aucun problème si chacun s'investit à peu près à la même mesure ; et c'est le cas dans bien des couples interrogés qui se ressemblent sur un point : ils ont adopté un fonctionnement souple. Un partage équilibré du travail à la maison ne veut pas dire que chaque partenaire participe à chacune des tâches. Patrice, 25 ans, enseignant, met la table et la débarrasse, fait la vaisselle, sort les poubelles et descend le matin chez le boulanger acheter pain et croissants. De son côté, Yves, 35 ans, cadre, prépare les repas, fait le ménage et s'occupe des lessives. Patrice ajoute qu'ils se sont répartis les tâches selon leurs «goûts et compétences », sans qu'il y ait eu négociation. En réalité, il accomplit certaines tâches qu'il juge peu agréables dans la mesure où son compagnon fait de même. La négociation ne passe, en effet, pas toujours par la parole ; elle peut s'avérer plus subtile, chacun adaptant son comportement en fonction des actes de son compagnon et du travail à effectuer. Ce témoignage soulève deux réflexions. D'abord la notion de «goûts et compétences » renvoie à une identité personnelle, en partie indépendante de l'identité sexuée ; les manières de faire ne seraient pas seulement féminines ou masculines, d'autres facteurs entrant dans leur adoption. La deuxième concerne la question du «miraculeux » hasard qui 
permettrait que les «goûts et compétences» de l'un et de l'autre s'accordent aussi parfaitement. Penchons-nous à présent sur des situations plus inégalitaires.

\section{Quand la répartition est inégale}

Agé de 30 ans, aide-soignant, Emmanuel vit depuis deux ans et demi avec Denis, 35 ans, cadre. Emmanuel écrit d'abord que la répartition des tâches n'a pas été définie et que leur organisation est souple, une affirmation contredite lorsqu'il entre dans le détail. Lui-même assume en fait la plupart des tâches ménagères, son compagnon s'occupant ponctuellement des travaux de bricolage et des plantes. Peut-être Emmanuel ne lui permet-il pas vraiment de s'investir plus, cette répartition n'étant pas pour lui source de mécontentement. Faire la cuisine lui plaît car il ne s'agit pas simplement de préparer de quoi se nourrir, mais surtout de préparer un moment important pour l'unité du couple. S'occuper des tâches ménagères n'est pas une corvée pour lui car elles contribuent au bien-être conjugal. Comment expliquer ces dispositions divergentes au regard des tâches ménagères ? Sans doute l'explication est-elle en partie à chercher dans une socialisation différente pour l'un et l'autre. Emmanuel dit que Denis, très jeune, aidait son père artisan sur des chantiers. Tourné très tôt vers les activités extérieures, conformément au modèle masculin, Denis ne serait pas disposé à prendre en charge des tâches ménagères. Dès l'enfance, Emmanuel a, quant à lui, dû prendre des responsabilités : aller chercher sa sœur à la maternelle, faire fonctionner les appareils ménagers dès six ans ; il dit avoir reçu une « éducation de type maternel ». Il tient à souligner la singularité d'une enfance pas comme les autres, singulière par le caractère exceptionnel des responsabilités à connotation féminine, tournées vers l'espace domestique. Plus loin, à propos de la répartition des tâches domestiques dans son couple, Emmanuel fait lui-même le lien entre ses pratiques conjugales d'aujourd'hui et ce qu'il a appris dans son enfance. On peut supposer que l'expérience d'Emmanuel et celle de quelques autres interlocuteurs, a eu une influence sur leur représentation du travail domestique : ces hommes sont plus souples et s'investissent plus volontiers dans les tâches ménagères, parfois jusqu'à en accepter la plus grande part. Ne se disant à aucun moment malheureux de cette répartition, ils semblent assumer sans problèmes des manières de faire traditionnellement réservées aux femmes.

À l'inverse, Tanguy, 34 ans, dessinateur, vit depuis dix ans avec Paul, 38 ans, animateur, et témoigne d'une organisation qui ne le satisfait pas. Au quotidien, il s'occupe du travail à la maison, et Paul, du travail à l'extérieur. Le soir, c'est Paul qui fait la cuisine, mais c'est Tanguy qui prépare et dessert la table. L'investissement de Tanguy dans le bricolage, qui ne relève pas de ses compétences, est tout à fait ponctuel. Comment Tanguy explique-t-il ce partage ? Il explique d'abord que «l'organisation s'est faite en fonction de (leurs) tendances naturelles » et précise ensuite qu'il provoque parfois des discussions au sujet de la négligence de Paul pour le ménage ou la vaisselle. Une contradiction est ainsi dévoilée : l'organisation des travaux domestiques n'a pas été définie en fonction des «goûts » et « dégoûts » de chacun mais en fonction de ceux de Paul. Comme il aime cuisiner, c'est lui qui prépare les repas ; comme il déteste faire le ménage et le repassage, c'est Tanguy qui s'en charge. Tanguy se trouve donc en charge des tâches ménagères qui sont celles qui causent le plus d'insatisfaction [Brousse, 2000].

\section{La question délicate de la saleté et du désordre}

Les tâches ménagères liées au traitement du sale et du désordonné sont systématiquement présentées par nos interlocuteurs comme étant à l'origine de discussions. Pourquoi ? On peut d'abord penser que s'occuper de la saleté correspond à un souci d'hygiène. Certes, mais comme nous y invite Mary Douglas, essayons de nous défaire de cette notion récente. D'abord «la saleté absolue n'existe pas »: elle n'est que relative par rapport à la perception que chacun a d'un certain ordre [Douglas, 2003 : 55]. Le premier malentendu dans les couples 
gays et dans les autres vient de là : chacun n'a pas la même perception que son compagnon de ce qui est sale ou en désordre, et n'agit donc pas toujours comme son compagnon l'attendrait. Ainsi Jean, 40 ans, gérant de société, ne range jamais sa tasse de café qui reste sur la table basse jusqu'au retour de son compagnon, David, 30 ans, aide-soignant, qui le lui a reproché « au moins cent fois ». De ce point de vue, la vie sous le même toit est un paradoxe : deux individus cohabitent avec deux perceptions différentes de l'ordre et du désordre, du propre et du sale, deux volontés de rendre le décor « conforme à (son) idée » [Douglas, 2003 : 24]. Les discussions, que David provoque régulièrement avec Jean, concernent systématiquement la vaisselle et le linge sale. Si le linge est porteur d'une mémoire féminine [Kaufmann, 1992], on peut supposer que cette mémoire féminine s'étend à l'ensemble du rapport à la propreté et que c'est là un point récurrent des difficultés rencontrées par les hommes en matière de travail domestique. La division du travail entre les sexes qui est encore, nous l'avons vu, inégalitaire, et qui repose sur une socialisation différenciée des garçons et des filles, ne favorise pas la prise en charge par les hommes des tâches liées au nettoyage de la saleté.

Alors que la répartition des charges financières adoptée par les couples gays est relativement stable, la norme égalitaire est plus diversement mise en œuvre dans le domaine du travail domestique. Sans doute parce qu'on touche ici aux représentations sociales imaginaires de ce qui fait un homme. Allons un peu plus loin dans la réflexion et c'est un de nos interlocuteurs qui nous indique cette voie. La question de l'identité sexuée traverse tous les domaines d'interaction au sein du couple, mais d'abord et surtout celui de la sexualité. Venant de dire qu'il assumait la plus grande part des tâches domestiques, il s'est écrié lors d'un entretien: «N'allez pas vous imaginer que c'est pour autant moi qui fait la femme! »

Sexualité conjugale : «Faire la femme »?

Cette expression montre le lien possible, souvent fait, entre l'organisation ménagère et la sexualité conjugale. Une sexualité conjugale gay présente quelques spécificités. En effet, les couples d'hommes pratiquent plus que les autres l'extra-conjugalité [Adam, 1999 : 58] ; notre enquête confirme l'importance de cette conjugalité ouverte à des rencontres occasionnelles. Néanmoins, s'ils sont soumis à la norme d'une sexualité multiple et récréative, les couples gays sont également traversés par l'idéal d'une conjugalité exclusive fortement valorisée dans notre société qui reste une sorte d'idéal de vie pour une majorité d'entre eux [Worth, 2002 : 243]. L'influence de ces deux normes contradictoires [Lerch, 2002] s'applique diversement selon les couples et les moments dans l'histoire conjugale. Mais, presque tous font en sorte que les rencontres ne soient qu'éphémères et ne concurrencent pas la dyade conjugale. Par ailleurs, cette pratique de l'extra-conjugalité reste marquée par une forte exigence d'équité. Personne ne veut être laissé pour compte. Devant le besoin de leur compagnon de faire des rencontres parallèles, certains hommes peu versés dans la sexualité récréative, se mettent eux aussi à avoir des aventures. D'autres, soucieux de partager l'exotisme d'un troisième partenaire et de limiter les vagabondages solitaires, expérimentent les ébats en trio. L'extraconjugalité répond au souci de son propre bonheur, mais elle ne doit pas contrevenir à l'équilibre conjugal, peut-être doit-elle y contribuer. On voit que les pratiques sexuelles conjugales elles-mêmes sont un champ marqué par la norme égalitaire. Comme les hétérosexuels aujourd'hui [Bozon, 2001a], les gays en couple ne sont pas forcément attachés à l'orgasme simultané ; la réciprocité peut donc être différée. Cependant, la plupart restent attachés à l'attention réciproque des désirs de chacun : elle peut s'exprimer dans des pratiques mutuelles simultanées ou différées (le rapport oral-génital simultané est souvent présenté comme la pratique la plus égalitaire), ou non. Par exemple Olivier préfère pratiquer la sodomie insertive et François, la sodomie réceptive : ils n'inversent donc jamais cette position 
et cela convient à chacun d'eux, d'autant plus qu'ils ont par ailleurs une relation plutôt égalitaire.

\section{Représentations de la pénétration anale}

La pénétration anale concerne la quasi-totalité des couples interrogés contrairement à la sexualité «de récréation» hors couple où elle intervient bien moins [Adam et al., 2002]. A l'instar de la pénétration vaginale que les femmes hétérosexuelles considèrent comme «la manifestation la plus concrète du lien et du rapprochement des partenaires » [Bozon, 2001a : 254], la pénétration anale est perçue par nombre de gays, comme une pratique étroitement liée au sentiment amoureux, ou du moins réservée au partenaire régulier [Mendes-Leite, 2003 : 213]. Or, cette pratique est fortement connotée comme masculine ou féminine selon le rôle « actif » ou «passif» : un homme, du fait de sa position pendant le coït anal, peut donc se voir classé du côté féminin (ibid.: 204) et par là du dominé [Bourdieu, 1998 : 27 ; Bozon, 2001b: 173; Hamel, 2003: 505]. Attribuées à un homme, des qualités typiquement féminines dans les représentations ont une valeur négative [Melhuus, $2001: 214]$; d'ailleurs des mots très péjoratifs qualifient dans de nombreuses sociétés les hommes pénétrés ${ }^{3}$. Malgré l'intérêt nouveau de certains hommes hétérosexuels pour la sodomie réceptive [Welzer-Lang, 2004 : 247], l'asymétrie des rôles et des places dans la sexualité est encore très ancrée dans les représentations ; elle trouve un contre-poids dans les pratiques qui favorisent la réciprocité et l'égalité. Jouer le rôle passif à tour de rôle dans une relation conjugale est perçu comme «plus démocratique, une preuve d'amour pour son partenaire » [Carballo-Dieguez, 2004 : 165]. C'est la raison pour laquelle l'alternance des rôles dans la sexualité conjugale est vigoureusement valorisée dans le discours de nos interlocuteurs, même si dans la pratique, certains hommes plus indépendants à l'égard des injonctions de la norme masculine, s'épanouissent tout en ayant un rôle exclusivement passif au regard de la sodomie.

\section{L'expérience d'une sexualité ressentie comme fortement inégalitaire}

Dans les couples hétérosexuels, une sexualité peu égalitaire fait écho à une répartition des tâches et des responsabilités peu équitables [Bozon, 1998 : 224-225]. Pour les couples gays, une répartition déséquilibrée des tâches ménagères pose d'importantes difficultés quand elle s'accompagne d'une organisation de la sexualité qui ne prend pas en compte les désirs des deux partenaires. En plus d'assumer la quasi intégralité des tâches ménagères, certains des hommes interrogés se voient assigner, pendant le coït anal, un rôle passif. Ce redoublement systématique de rôles qu'ils se représentent comme "féminins » leur pose un problème d'identité de sexe. C'est le cas de Michel, 36 ans, cadre, qui vit depuis dix-sept ans avec JeanMarc, 44 ans, cadre et élu local. Lors de notre première rencontre, Michel déclare avoir en charge les tâches ménagères, mais cela ne lui pose «aucun problème ». Lors d'un second entretien, Michel confie que sa sexualité avec Jean-Marc ne le « rend pas heureux »: "Je ne peux pas être actif. J'ai essayé, mais Jean-Marc ne veut pas. Il dit qu'il ne peut pas ». Au début, cette situation ne le gênait pas. Mais au fil des années, Michel, qui, notons-le, a huit ans de moins que son compagnon, avoue ressentir «un manque, une frustration » dans le fait d'être cantonné à un rôle sexuel qu'il vit aujourd'hui difficilement. En conséquence, Michel s'apprête à remettre en cause la fidélité à laquelle tous les deux s'étaient engagés. Il aimerait faire des rencontres sexuelles qui combleraient ses attentes. Outre les raisons physiologiques invoquées par Jean-Marc, qui l'empêcheraient d'être "passif », on peut supposer que la part de féminité identifiée à la passivité sexuelle est en cause. Le refus d'interversion des rôles et le manque d'attention à ses désirs ressenti par Michel, sont pour lui des signes négatifs de leur lien amoureux. Cette situation est d'autant plus douloureuse qu'elle se renouvelle dans le champ du travail domestique. 


\title{
Un cas de violence conjugale
}

Revenons au témoignage de David. Le fait qu'il assume l'intégralité des tâches ménagères, alors même que Jean y semble indifférent, est à l'origine de bien des disputes qui parfois dégénèrent. Il confie que leur relation est conflictuelle depuis un an: "c'est un truc vachement dur parce que j'ai connu un côté de Jean que je ne connaissais pas, qui peut être extrêmement violent... d'une violence à te faire taper dessus. Si tu veux, on a déjà eu deux, trois petites bagarres où j'ai pris des coups ». Le témoignage de David est unique dans notre enquête. Du mode de répartition des tâches ménagères à ces disputes effroyables au cours desquelles il reçoit des coups violents, David cumule des positions caractéristiques du poids de la domination masculine : il a dix ans de moins que son ami, s'occupe de la totalité des tâches ménagères, se trouve assigné à un rôle sexuel passif et a été victime de coups. La souffrance ressentie par David au moment où il livre son histoire prend sa source tant dans la violence des disputes que dans le cumul de rôles synonymes de soumission. Alors comment se vivre à égalité avec son compagnon quand tant de choses dans sa vie de couple mettent à mal l'image de sa propre identité masculine?

L'iniquité conjugale : un modus vivendi insupportable pour les couples gays

Bien que les couples gays, ainsi que les autres couples, posent comme principe l'égalité entre les amants - égalité qui serait plus facile à mettre en œuvre entre deux hommes qu'entre un homme et une femme -ils vivent, non sans difficultés, des situations conjugales qui mettent à l'épreuve le principe proclamé. Le modèle hiérarchique des relations entre les sexes semble, en effet, traverser tous les couples, affectant négativement des activités, des gestes ou des situations corporelles connotées comme féminines. Parce que la plus grande partie des hommes a eu une socialisation typiquement masculine, un homme en couple avec un autre homme ne peut se voir assigné sans difficulté à des champs de compétences domestiques, et à des rôles traditionnellement perçus comme féminins et subalternes. La position de celui qui est renvoyé du côté du féminin et du dominé est d'autant plus insupportable qu'elle cumule les inégalités, en particulier dans le domaine le plus sensible, celui de la sexualité anale passive non réciproque.

\begin{abstract}
ANNEXE
Cette enquête a été réalisée en France entre 2000 et 2005, avec le soutien de l'Agence nationale de recherche sur le sida dans le cadre d'une thèse en anthropologie sociale. 34 hommes ont répondu à un texte d'invite qui faisait appel à des gays pour évoquer leur vie de couple. Répondaient ceux qui se reconnaissaient dans les expressions « couple gay » et «vie de couple». Les entretiens ne permettant pas toujours d'aborder les domaines les plus intimes de la vie conjugale, nous avons choisi une méthodologie diversifiée. Supposant que l'écrit serait un canal plus favorable pour aborder l'intimité [Le Gall, Le Van, 1999], la plupart des contacts ont été pris par le biais d'internet. Certains de nos interlocuteurs nous ont envoyé un récit de vie via internet, suivi d'un échange par mail, parfois aussi de conversations ${ }^{4}$ en face-à-face, ou d'un entretien téléphonique. L'anonymat des relations par internet introduit à la fois distance et proximité. L'enquêteur ne fait alors plus figure d'étranger pour devenir le temps du témoignage (plusieurs semaines sinon plusieurs mois) quelqu'un de proche et de lointain à la fois. En parallèle, l'enquête repose sur l'observation d'un couple, pendant plusieurs années.

La méthode de recueil des données introduit un biais quant aux caractéristiques des hommes interrogés. Ils habitent dans toute la France, souvent en ville, voire dans une métropole régionale et quelquefois en milieu rural. Les catégories socio-professionnelles sont le plus souvent intermédiaires et les enquêtés ont tous un accès relativement facile à l'écrit. Ils ont de 24 à 50 ans, mais les trentenaires sont sur-représentés. Les spécificités du corpus tiennent aussi aux caractéristiques de la population homosexuelle masculine [Schiltz, 1997].
\end{abstract}

\section{Références bibliographiques}


ADAM Philippe, 1999, "Bonheur dans le ghetto ou bonheur domestique ? Enquête sur l'évolution des expériences homosexuelles », Actes de la Recherche en Sciences Sociales, $128: 56-72$.

ADAM Philippe, Eric HAUET, Caroline CARON, 2002, Recrudescence des prises de risque et des MST parmi les gays. Résultats préliminaires de l'enquête Presse gay 2000, INVS, DGS, ANRS.

ANXO Dominique, Lennart FLOOD, Yusuf KOCOGLU, 2002, «Offre de travail et répartition des activités domestiques et parentales au sein du couple : une comparaison entre la France et la Suède », Economie et Statistique, 352 : 127-150.

BARRERE-MAURISSON Marie-Agnès, 2003, Travail, famille : le nouveau contrat, Paris, Gallimard.

BOURDIEU Pierre, 1998, La domination masculine, Paris, Le Seuil.

BOZON Michel, 1998, «Amour, désir et durée. Cycle de la vie conjugale et rapports entre hommes et femmes », in Bajos Nathalie, Bozon Michel, Ferrand Alexis, Giami Alain, Spira Alfred, La Sexualité aux temps du sida, Paris, PUF : 175-234.

2001a, «Sexualité et conjugalité », in Bloss Thierry (dir.), La dialectique des rapports hommes-femmes, Paris, PUF : 239-259.

- 2001b, «Sexualité et genre », in Laufer Jacqueline, Marry Catherine, Maruani Margaret (dir.), Masculin-Féminin : questions pour les sciences de l'homme, Paris, PUF : 169-186.

BROUSSE Cécile, 2000, «La répartition du travail domestique entre hommes et femmes », Dossiers et Recherches, INED, 84 : 89-106.

CARBALLO-DIEGUEZ Alex, Curtis DOLEZAL, Luis NIEVES, Francisco DIAZ, Carlos DECENA, Ivan BALAN, 2004, «Looking for a tall, dark, macho man... Sexual-role behaviour variations in Latino gay and bisexual men », Culture, Health \& Sexuality, 6-2 : 159-171.

CARRINGTON Christopher, 1999, No Place Like Home : Relationships and Family Life among Lesbians and Gay Men, Chicago, University of Chicago Press.

DOUGLAS Mary, 2003 (1967), De la souillure. Essai sur les notions de pollution et de tabou, Paris, La Découverte.

DUSSUET Annie, 1997, Logiques domestiques, Paris, L'Harmattan.

FILIOD Jean-Paul, 2003, Le désordre domestique. Essai d'anthropologie, Paris, L'Harmattan.

HAMEL Christelle, 2003, L'intrication des rapports sociaux de sexe, de "race ", d'âge et de classe: ses effets sur la gestion des risques d'infection par le VIH chez les Français descendant de migrants du Maghreb, Thèse de doctorat en anthropologie, Paris, EHESS.

KAUFMANN Jean-Claude, 1992, La trame conjugale. Analyse du couple par son linge, Paris, Nathan.

KELLERHALS Jean, Eric WIDMER, René LEVY, 2004, Mesure et démesure du couple. Cohésion, crises et résilience dans la vie des couples, Paris, Payot.

LE GALL Didier, LE VAN Charlotte, 1999, « Le premier rapport sexuel. Les mots pour ne pas le dire », Bastidiana, Sexualité et Sociétés, 27-28 : 121-139.

LERCH Arnaud, 2002, «Les éthiques conjugales gaies à l'épreuve du multipartenariat », in Lagrave Rose Marie, Gestin Agathe, Lépinard Eléonore, Pruvost Geneviève (dir.), Dissemblances. Jeux et enjeux du genre, Paris, L'Harmattan : 65-78.

MELHUUS Marit, 2001, "The power of penetration - The value of virginity : male and female in Mexican heterosexual and homosexual relations », in Catherine Alès, Cécile Barraud (dir.), Sexe relatif ou sexe absolu?, Paris, Editions MSH : 201-227.

MENDES-LEITE Rommel, 2003, «Sens et contexte dans les recherches sur les (homo) sexualités et le sida: réflexions sur le sexe anal », in Christophe Broqua, France Lert, Yves 
Souteyrand (dir.), Homosexualités au temps du sida. Tensions sociales et identitaires, Paris, CRIPS, ANRS : 199-220.

OLIVIER DE SARDAN Jean-Pierre, 1995, «La politique du terrain. Sur la production des données en anthropologie », Enquête, 1 : 71-109.

POLLAK Michael, 1982, "L'homosexualité masculine ou le bonheur dans le ghetto ?», Communications, $35: 56-80$.

SCHILTZ Marie-Ange, 1997, « Parcours homosexuels : une sexualité non traditionnelle dans des réseaux d'échanges sexuels à forte prévalence du VIH », Population, 52-6 : 1485-1536.

- 1998, «Un ordinaire insolite : le couple homosexuel », Actes de la Recherche en Sciences Sociales, 125 : 30-43.

WELZER-LANG Daniel, 2004, Les hommes aussi changent, Payot, Paris.

WORTH Heather, Alison REID, Karen MCMILLAN, 2002, " Somewhere over the rainbow : love, trust and monogamy in gay relationships », Journal of Sociology, $38: 237-253$.

\footnotetext{
134 hommes ont témoigné pour cette enquête. 7 d'entre eux ont moins de 2 ans de différence avec leur compagnon, 10 entre 3 et 5 ans, 8 entre 6 et 8 ans, 4 entre 9 et 15 ans. 16 couples disposent des revenus identiques; pour les autres, les différences de revenus sont variables, entre 300 et 1000 euros. 13 couples sont dans des situations socio-professionnelles très dissymétriques, qui recoupent le plus souvent les différences d'âge et de revenus. Cf. Annexe en fin d'article pour la méthodologie.

${ }^{2}$ La question de la gestion des ressources dans les couples gays est approfondie dans une contribution à l'ouvrage « La valeur des liens. Hommes, femmes et comptes familiaux », dirigé par Agnès Martial, à paraître en 2006.

${ }^{3}$ Des termes relevant du langage courant ou argotique assignent les hommes passifs à une place féminine et ont une dimension péjorative : « maricon » au Mexique, «bicha » au Brésil.

${ }^{4}$ Jean-Pierre Olivier de Sardan parle de l'entretien comme conversation, « une stratégie récurrente de l'entretien ethnographique, qui vise justement à réduire au minimum l'artificialité de la situation d'entretien » [Olivier de Sardan, 1995].
} 